\title{
Improved Rapid Automatic Keyword Extraction for Voice-based Mechanical Arm Control
}

\author{
Chi-Huang Shih, ${ }^{1}$ Cheng-Jian Lin, ${ }^{1,2^{*}}$ and Shiou-Yun Jeng ${ }^{3}$ \\ ${ }^{1}$ Department of Computer Science and Information Engineering, National Chin-Yi University of Technology, \\ Taichung 411, Taiwan \\ ${ }^{2}$ College of Intelligence, National Taichung University of Science and Technology, Taichung 404, Taiwan \\ ${ }^{3}$ Department of Business Administration, Asia University, Taichung 413, Taiwan
}

(Received January 11, 2021; accepted May 27, 2021)

Keywords: mechanical arm control, speech recognition, word segmentation, keyword extraction

As smart voices gradually enter the human living environment, people can interact with controlled systems through simple commands without the need for a screen. Even people without specialist training can use voice sensing control to easily complete the operation of a robot arm while avoiding incorrect operation caused by insufficient professional knowledge. In this study, we use the Google speech recognition engine to convert input speech into text information, and then perform speech recognition, word segmentation, and part-of-speech (POS) tagging. We also propose an improved rapid automatic keyword extraction (IRAKE) method, which uses the word string matching feature in the dictionary method to correspond to the relevant execution action function. The experimental results show that the accuracy rate of the proposed IRAKE method is $16 \%$ higher than that of the traditional rapid automatic keyword extraction (RAKE) method.

\section{Introduction}

In recent years, with the maturation of speech recognition technology, its application fields have become extensive and include common speech processing tasks such as machine translation, ${ }^{(1)}$ question answering, ${ }^{(2)}$ and sentiment analysis. ${ }^{(3)}$ The related technologies of speech processing include machine learning, data mining, knowledge acquisition related to language processing, and linguistic research related to language computing. However, most existing speech recognition ${ }^{(4,5)}$ and natural language processing ${ }^{(2,6)}$ systems do not involve the control of industrial equipment. This is because reliable systems are seldom used in real industrial environments, rather than because of the inadequacy of the technology or the unsuitability of the voice sensing control system for industrial control. An industrial voice sensing control system must meet specific requirements that are more stringent than those in non-industrial situations before it can be used in production lines. Before executing these instructions, the voice assistant system must first understand the human language, so speech recognition and processing technologies for natural language understanding ${ }^{(7,8)}$ are particularly important.

*Corresponding author: e-mail: cjlin@ncut.edu.tw

https://doi.org/10.18494/SAM.2021.3266 
Recently, many researchers ${ }^{(9-13)}$ have used various methods for implementing voice sensing control. Kennington and Schlangen ${ }^{(9)}$ used Markov logic networks to enhance natural language understanding. Wang et al. ${ }^{(10)}$ and Savage et al. ${ }^{(11)}$ used Microsoft SAPI for voice sensing control systems. Rada and Paul ${ }^{(12)}$ proposed the TextRank method based on graph sorting to extract keywords in text. The idea of this method is to design a formula through a voting idea inspired by Google's PageRank algorithm. This method also considers the relationship between the meanings of adjacent words and adds text weights. After iteratively calculating the rank value of each node and sorting, the keywords are obtained. Although this method has good results for Chinese extraction, it is less suitable for keyword extraction in English. In addition, Kumar and Zayaraz ${ }^{(13)}$ and Mohasseb et al. ${ }^{(14)}$ proposed a method of extracting the conceptual relationship from a question and answer system through a classifier and classifying the questions into a predefined list of answer types, where candidate answers are screened to improve classification accuracy. Although research on question and answer type classification is not new, designing effective methods is still a challenging task. The main reason is that the question and answer sentences are usually very concise, and just one sentence can clarify the meaning of a question.

Although the above methods can be applied in certain fields, the problems associated with their application to industrial voice sensing control systems are still difficult to solve. In this study, we use the Google speech recognition engine to convert input speech into text information, and then perform speech recognition, word segmentation, and part-of-speech (POS) tagging. We also propose an improved rapid automatic keyword extraction (IRAKE) method. In feature extraction, we use regular expressions and a variable storage method to solve the problem of numerical extraction in an industrial setting, and use a string matching method to convert strings into mechanical codes, and finally use the mechanical arm to perform the corresponding action.

The remaining sections of this paper are structured as follows. Section 2 introduces the background description and preprocessing of natural language. The original keyword extraction method and the improved keyword extraction method are described in Sect. 3. Section 4 reports the results of experimental and quantitative evaluations. Finally, a summary and future work are discussed in Sect. 5.

\section{Natural Language Processing}

Language is a good medium of communication. It is not only an art, but also a bridge of communication between people. When a voice assistant receives a command, through internal decoding and parsing, the voice is converted into a text message (i.e., speech to text), or a voiceassisted ideal expression is converted into a sentence understandable by humans (i.e., natural language generation). After the sentence enters the processing layer, word segmentation, part-ofspeech tagging, and keyword extraction can be performed according to the task. Then, the computer can understand the content of the sentence and communicate smoothly with humans. A flow chart of natural language processing is shown in Fig. 1. 


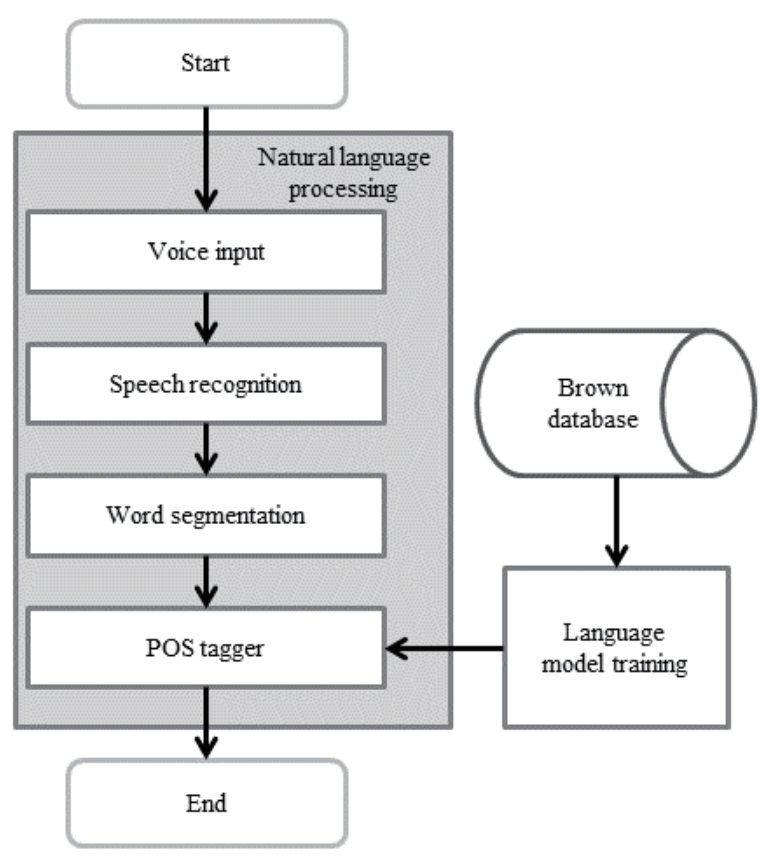

Fig. 1. Flow chart of natural language processing.

\subsection{Speech recognition}

Speech recognition has attracted increasing attention. The main purpose of studying speech recognition is to enable voice assistants to convert voice signals into corresponding text or messages through recognition and understanding. Its technical coverage is very extensive, including signal processing, acoustic modeling, linguistics, decoders, and other closely related disciplines. Since the Google Speech API module is widely used to realize real-time conversion of speech signals into text, we also use this technology in this study.

\subsection{Word segmentation}

Word segmentation is a basic and very important task in natural language processing, and its main purpose is to allow computers to process words and languages and convert them into natural languages that computers can understand. In linguistics, words are the smallest linguistic units that can be used independently and contain semantic content. Each word has a complete meaning. In English sentences, blank characters and punctuation marks are used as the basis for segmentation, as shown in Table 1.

\subsection{POS tagger}

A POS tagger is not only a method of analyzing sentence components, but also a procedure to mark a correct part of the speech for each word after the result of word segmentation. The labeling procedure is based on the role of the words in the syntactic structure, with each part of the sentence given the appropriate part of the speech. Its main purpose is to achieve effective 
Table 1

Output comparison results before and after word segmentation.

\begin{tabular}{lc}
\hline Input & go back ten millimeters \\
\hline Output & 'go', 'back', 'ten', 'millimeters' \\
\hline
\end{tabular}

Table 2

Word ambiguity resolution.

(1)

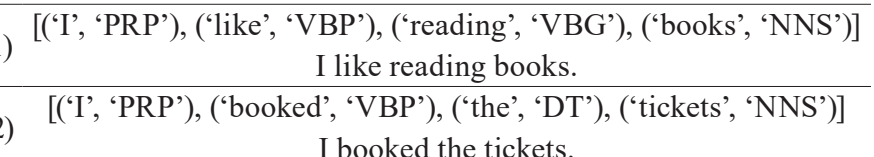

Table 3

Output comparison results before and after word segmentation.

\begin{tabular}{lccc}
\hline POS & POS classification & POS description & Examples \\
\hline CC & Conjunction & Coord conjunction & and, or, but \\
CD & Noun & Cardinal number & one, two \\
DT & Article & Determiner & the, some \\
IN & Preposition & Preposition & of, in, by \\
JJ & Adjective & Adjective & small \\
NN & Noun & Noun, sing. or mass & workpiece \\
VB & Verb & Verb, base form & move \\
RB & Adverb & Adverb & slowly \\
\hline
\end{tabular}

vocabulary classification and resolve ambiguity resolution problems through POS tagging. For example, the same word "book" has two different meanings, with the meaning differing with the placement. Here, we use the natural language toolkit for POS tagging, as shown in Table 2.

POS tagging is usually used for information extraction, proper noun tagging, and sentence structure analysis. The basic POSs can be divided into verbs, nouns, adjectives, and other categories, such as prepositions, conjunctions, and pronouns, as shown in Table 3.

\section{Proposed Method}

This section introduces the proposed IRAKE method of keyword extraction. We use the results of the natural language processing mentioned in Sect. 2 with the selection mechanism to find candidate keywords. Some of these candidate keywords may contain numerical or decimal point keywords. Here, we adopt a solution for effective access to numerical values, transfer the numerical values to the predefined format corpus, and then use unit conversion to obtain the actual moving distance of the mechanical arm. Finally, a dictionary method is used to match the mechanical code to control a mechanical arm. A flow chart of the overall system is shown in Fig. 2.

\subsection{Proposed IRAKE}

The rapid automatic keyword extraction (RAKE) algorithm was proposed by Rose et al. ${ }^{(15)}$ in 2010. For both processing text and answering questions, keyword extraction is very important 


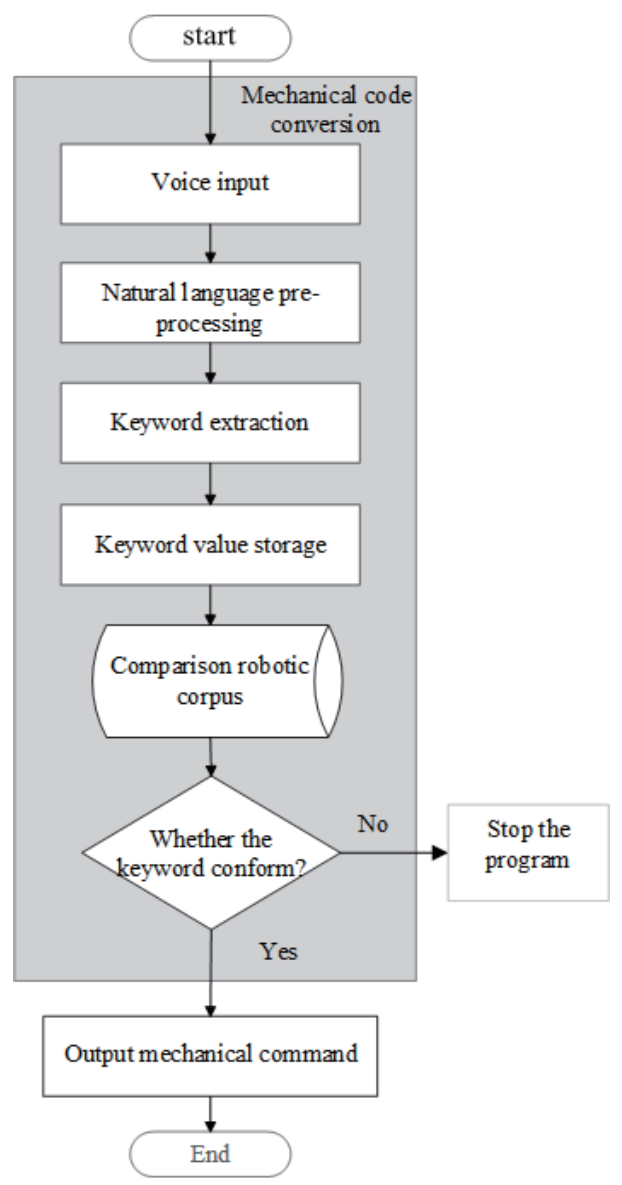

Fig. 2. Flow chart of mechanical code conversion.

for natural language processing. When the message reaches the robotic arm, keyword extraction can be used to effectively identify the key points of the content and execute the corresponding instructions to complete the task. To effectively extract English keywords, the algorithm in this study is used to extract keywords quickly and automatically for voice assistants. There are usually three main steps in the keyword extraction algorithm:

Step 1: Use punctuation marks (commas, periods, question marks, exclamation marks, etc.) to divide a document or sentence into several clauses.

Step 2: Extract keyword candidates through stop words. The extracted keywords may be one word or multiple words depending on the task.

Step 3: Select the score for each keyword by combining the properties of all candidate words into a formula to obtain a score. The formula for the word score $(S)$ is

$$
S=\frac{D}{F},
$$

where $F$ represents the frequency of the word in the sentence and $D$ represents the dimension of 
the word. In the process of finding $D$, in addition to the number of occurrences of each candidate keyword, the co-occurrence of the words that appear in each phrase is considered. When the word co-occurrence dimension appears once, it is incremented by 1 . Here, we input the original sentence "Please show the current dynamic error and static error." by voice as an example. Figure 3 shows a diagram of the co-occurrence of words in the text content.

After filtering in Steps 1 and 2, we obtain the candidate keywords "current dynamic error", "static error", and "please show". Then, Eq. (1) is used to calculate the score of each key candidate. The details are shown in Fig. 4 and Table 4.

Although the original fast and automatic keyword extraction method can effectively and quickly extract keyword candidates through punctuation and stop words, if the sentence contains a decimal value, the decimal point in the decimal value is regarded as punctuation for segmenting phrases. In addition, keyword candidates are extracted through stop words. The extracted keywords may be one word or multiple words. Although the above method can capture keywords, it is difficult to match the instructions in the future. For example, Table 5 shows that the decimal point in the decimal value 5.5 is regarded as punctuation for segmenting phrases. Therefore, the output of the keyword extraction is 5 .

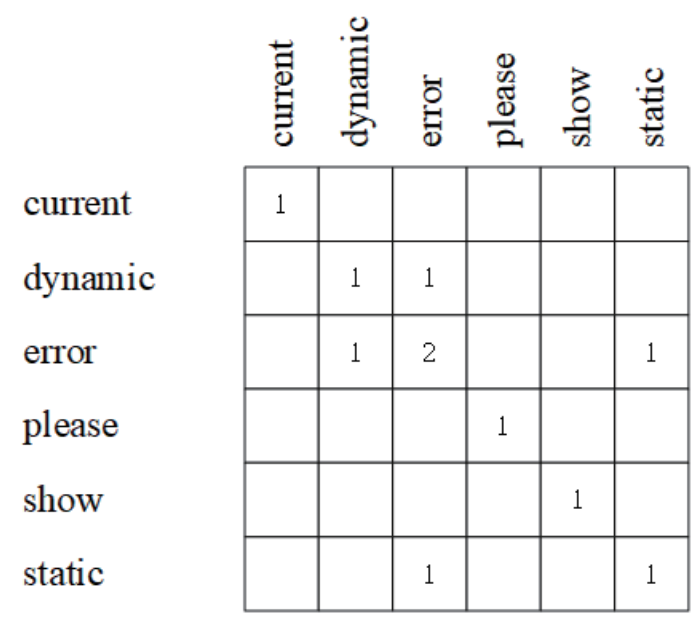

Fig. 3. Diagram of co-occurrence of words in text content.

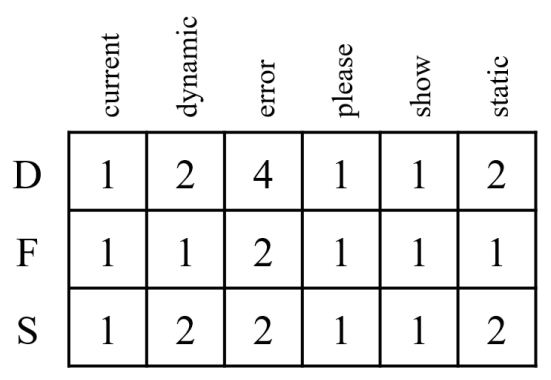

Fig. 4. Diagram of score calculation.

Table 4

Keyword outputs (voice input, word segmentation, keyword extraction).

\begin{tabular}{lr}
\hline Voice input & please show the current dynamic error and static error \\
\hline Word segmentation & ['please', 'show', 'the', 'current', 'dynamic', 'error', 'and', 'static', 'error'] \\
\hline Keyword extraction & ['current dynamic error', 'static error', 'please show'] \\
\hline
\end{tabular}

Table 5

Keyword extraction results of original RAKE.

\begin{tabular}{lr}
\hline Voice input & rotate 5.5 degree clockwise around the $\mathrm{x}$ axis \\
\hline Word segmentation & 'rotate', '5.5', 'degree', 'clockwise', 'around', 'the', 'x', 'axis' \\
\hline Keyword extraction & '5 degree clockwise around', 'rotate 5', 'x axis' \\
\hline
\end{tabular}


Therefore, the main purpose of this paper is to propose an IRAKE method to solve the problem of decimal points and format output. The proposed IRAKE method is described as follows. Introduce a regular expression in Step 1 of the original RAKE method to solve the decimal point problem. First, set some special words and character combinations in the regular expression, and then pass the combined regular word string to filter the expression. The specific content of the match is obtained as shown in Table 6.

In the format part, the Python split function is used. The input sentences are first used as spaces to divide the words, and it is judged whether the conditions in these sentences contain stop words. If there are stop words, then they are directly discarded; if not, the words are stored in an array. The output is shown in Table 7.

\subsection{Numerical storage representation of keywords}

When applying voice sensing control systems, the use of numerical values in the industrial field has been a very common problem. In particular, the numerical values cover a very large range in high-precision robotic arm applications. If all the numerical values are included in the corpus, the amount of data in the corpus will be greatly increased, making control difficult. Therefore, we use the following approach to effectively access the values. First, the sentence is preprocessed. Here, we use the word segmentation results in Sect. 2.2 to create a corpus and extract features according to the sentence format. When the input sentence contains numerical features, we extract them and transfer them to another variable, which is defined as a number. In this way, even if the input numerical value changes, it will not affect the subsequent corpus matching. Finally, this numerical value is extracted through the label. The results are shown in Table 8 .

Table 6

Regular expressions used in method for special words and character combinations.

\begin{tabular}{lcc}
\hline Grammar & Regular expression & Example \\
\hline Integer & {$[0-9]+$} & 1234 \\
Real number with decimal point & {$[0-9]+\backslash .[0-9]+$} & 0.25 \\
English vocabulary & {$[$ A-Za-Z]+ } & move \\
Variable name & {$[$ A-Za-Z_][A-Za-Z0-9_]* } & number \\
\hline
\end{tabular}

Table 7

Keyword extraction results using IRAKE method.

\begin{tabular}{lr}
\hline Voice input & rotate 5.5 degrees clockwise around the $\mathrm{x}$ axis \\
\hline Word segmentation & 'rotate', '5.5', 'degrees', 'clockwise', 'around', 'the', 'x', 'axis' \\
\hline Keyword extraction & 'rotate 5.5 degrees clockwise around x axis'
\end{tabular}

Table 8

Numerical storage representation of keywords.

\begin{tabular}{lllcl}
\hline Voice input & go & back & $\mathbf{1 . 5}$ & millimeters \\
\hline Corpus establishment & go & back & number & millimeters \\
\hline
\end{tabular}




\subsection{Unit value conversion}

Although a value can be effectively stored, if different unit values are encountered, the mechanical arm still has no way of understanding the distance the user wants it to move. The moving distance of the mechanical arm must be obtained through unit conversion. Here, two parameters (value and unit) are defined, which are assigned to specific variables, and a standard value of a unit is formulated as shown in Table 9.

\subsection{Instruction code conversion}

There are two common methods in implementing instruction code conversion. The first one is a dictionary method based on a database search, and the second one is a statistical method combined with machine learning and multiple algorithms.

(1) Dictionary method: This method uses a keyword-value pair to query the dictionary to determine whether it is a feature word. The feature word here means a mechanical code. The mechanical code is defined manually. After finding out some features, a network dictionary, such as WordNet, is used to search for synonyms, which are added to the dictionary. When querying commands, the dictionary is checked to determine whether each command is a feature word. The advantage of this method is that it is quick to use. It only needs to perform a manual judgment and then find the same word in the dictionary of synonyms. The disadvantage is a lack of diversity. If there are words in a command that are not in the dictionary, the system cannot perform the command.

(2) Statistical method: Machine learning is used in cooperation with statistics or other algorithms to query feature words. The advantage is that when training with data is completed, the feature words can be automatically predicted without manual screening. The disadvantage is that a manual search for a certain amount of training data and test data is required in advance. The quality of the training data will affect the accuracy of the test.

On the basis of the above analysis and discussion, the industrial cases are considered to be customized services, and the existing corpus is limited. Therefore, in this study, the dictionary method is used in the implementation. A schematic diagram of string matching in this method is shown in Fig. 5.

Table 9

Unit value conversion.

\#def distance move $*$ number value $*$ units:v1 $* \mathrm{v} 2$

\#def number value

fifteen: 15

sixteen: 16

seventeen:17

\#def units

millimeters:0.001

centimeters: 0.01

meters:1 


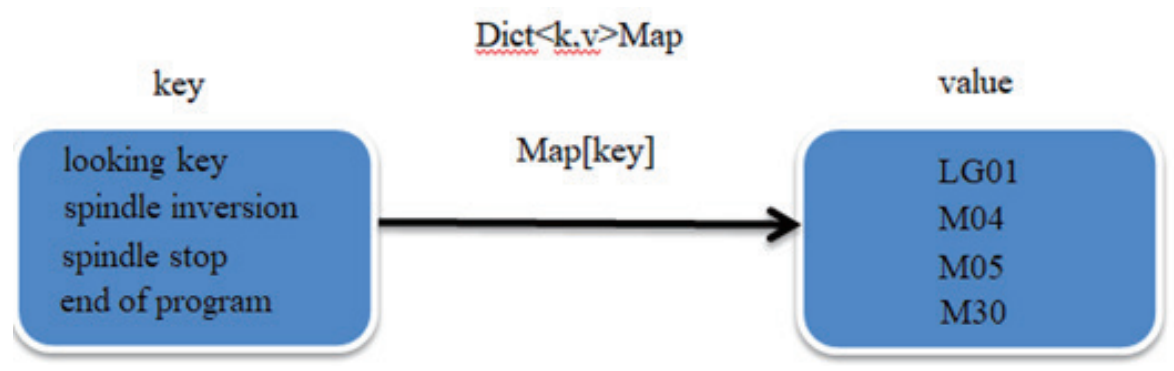

Fig. 5. (Color online) Schematic diagram of string matching.

\begin{tabular}{|l|}
\hline \multicolumn{1}{|c|}{ Keyword establishment in corpus } \\
\hline machine moves quickly x 100.0 y $123 \mathrm{z} 0.5$ \\
end program \\
Machine feed rate per minute num \\
Machine operation stopped num \\
Tool wear replacement num \\
workpiece made num pieces \\
looking key \\
looking Circle \\
looking rectangle \\
emergency stop \\
\hline
\end{tabular}

\begin{tabular}{|c|}
\hline Label \\
\hline 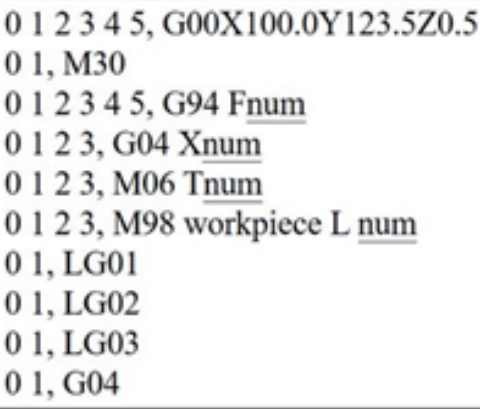 \\
\hline
\end{tabular}

Fig. 6. (Color online) Tag code conversion of keywords in corpus.

\begin{tabular}{|l|}
\hline \multicolumn{1}{|c|}{ Mechanical code } \\
\hline G00X100.0Y123.5Z0.5 \\
M30 \\
G94 Fnum \\
G04 Xnum \\
M06 Tum \\
M98 workpiece L num \\
LG01 \\
LG02 \\
LG03 \\
G04 \\
\hline
\end{tabular}

Fig. 7. Mechanical code conversion results.

First, two corpora are created in the back-end part, which are a keyword corpus and a mechanical code corpus, and then each keyword is tagged. The concept of string matching in the dictionary method is used to quickly look up the table to determine whether a string is a characteristic word. The tag code conversion of keywords in the corpus is shown in Fig. 6.

If the command format of the mechanical arm is converted using the proposed IRAKE method of keyword extraction, the corresponding machine codes will be obtained. These codes are the instructions to the mechanical arm. The mechanical code conversion results are shown in Fig. 7. 


\section{Experimental Results}

To verify the effectiveness of the proposed improved keyword extraction method, we use the keywords of the mechanical arm provided by the manufacturer plus a self-built corpus as the evaluation standard. Two types of voice command delivery are considered in the experiment, Google voice delivery and human voice delivery.

Google voice delivery mainly transmits commands to the robot arm, which are transferred to the voice assistant after Google translates them in advance. Therefore, it can effectively receive messages and execute corresponding commands. Human voice delivery is more concise and intuitive than Google voice delivery. The user can directly exercise control commands to the voice assistant through the microphone. When the voice assistant receives the message, it can also execute the corresponding mechanical arm motion.

In this keyword extraction experiment, we use 100 sentences that we accumulated as test data, and ten people are employed to evaluate the accuracy of keyword extraction. The experimental results obtained using the proposed method are compared with those obtained with other methods. ${ }^{(15,16)}$

\subsection{Results of keyword extraction}

To verify the effectiveness of the proposed IRAKE method, we compare it with the original RAKE method ${ }^{(15)}$ and the traditional keyword extraction method (TF-IDF). ${ }^{(16)}$ Precision $(P)$, recall $(R)$, and $f$-score are used as quantitative indicators to evaluate performance, where

$$
\begin{gathered}
P=\frac{T P}{T P+F P}, \\
R=\frac{T P}{T P+F N}, \\
f \text {-score }=\frac{2 P R}{P+R}=\frac{2 T P}{2 T P+F P+F N} .
\end{gathered}
$$

Here, $T P$ (true positive) represents the model correctly predicting the positive extracted keyword, $T N$ (true negative) represents the model correctly predicting the negative extracted keyword, $F P$ (false positive) represents the model incorrectly predicting the positive extracted keyword, and $F N$ (false negative) represents the model incorrectly predicting the negative extracted keyword. Precision represents the percentage of these extracted keywords that are correctly predicted. Recall represents the percentage of correct extraction among all the correct keywords. The $f$-score is a combination of precision and recall.

Tables 10 and 11 show a quantitative comparison of Google voice delivery and human voice delivery obtained in the experiment using various methods, respectively. It can be seen that the accuracy obtained by translation by Google in advance is $10 \%$ higher than that obtained by 
Table 10

Quantitative comparison of Google voice delivery using various methods.

\begin{tabular}{lccc}
\hline Method & Precision & Recall & $f$-score \\
\hline TF-IDF $^{(16)}$ & 0.71 & 0.89 & 0.80 \\
RAKE $^{(15)}$ & 0.63 & 0.77 & 0.70 \\
Proposed IRAKE & 0.79 & 0.93 & 0.86 \\
\hline
\end{tabular}

Table 11

Quantitative comparison of human voice delivery using various methods.

\begin{tabular}{lccc}
\hline Method & Precision & Recall & $f$-score \\
\hline TF-IDF $^{(16)}$ & 0.59 & 0.79 & 0.69 \\
RAKE $^{(15)}$ & 0.51 & 0.73 & 0.62 \\
Proposed IRAKE & 0.68 & 0.82 & 0.75 \\
\hline
\end{tabular}

Table 12

Comparison of results of keyword extraction for sentence containing a number by various methods.

\begin{tabular}{lc}
\hline Method & Keyword extraction results \\
\hline TF-IDF & rotate 10 degrees clockwise around axis \\
RAKE & "rotate 10 degrees clockwise around", "x axis" \\
Proposed IRAKE & rotate 10 degrees clockwise around x axis \\
\hline
\end{tabular}

Table 13

Comparison of results of keyword extraction for sentence containing decimal by various methods.

\begin{tabular}{lc}
\hline Method & Keyword extraction results \\
\hline TF-IDF & rotate 5.5 degrees clockwise around axis \\
RAKE & "5 degrees clockwise around", "rotate 5", "x axis" \\
Proposed IRAKE & rotate 5.5 degrees clockwise around x axis \\
\hline
\end{tabular}

manually sending commands to the voice assistant. The main reason is that the translation through Google in advance is much more stable than human voice delivery in terms of syllables, timbre, and pronunciation.

Next, to verify that the proposed improved keyword extraction method is superior to other methods, we select two more challenging sentences from the 100 -sentence corpus for discussion and analysis. For example, we enter the following sentence containing a number: "rotate 10 degrees clockwise around $\mathrm{x}$ axis".

A comparison of the experimental results of keyword extraction for this sentence containing a number obtained by various methods is shown in Table 12. Although the original RAKE method can effectively capture English words, the output format makes it difficult to transfer the command, and a format conversion is required. Although TF-IDF has no format conversion problem, it cannot effectively extract English words.

To meet the needs of industrial application, unit movements are calculated in $\mathrm{cm}$ and $\mathrm{mm}$, and the above example is slightly modified. In the numerical part, the original integer value is modified to a decimal. The testing sentence is changed to "rotate 5.5 degrees clockwise around $\mathrm{x}$ axis". A comparison of the results of keyword extraction for the sentence containing a decimal by various methods is shown in Table 13. When the original RAKE method encounters the decimal point, it uses the decimal point as a punctuation mark to segment the sentence into short expressions and shuffles their order. The proposed IRAKE method has better results for 
keyword extraction, making it beneficial for communicating with a mechanical arm via numerical values.

\section{Conclusions}

In this study, the proposed IRAKE method is applied to the voice sensing control of a mechanical arm. This method mainly extracts the numerical values of keywords using regular expressions, and extracts keyword candidates using the stop word filtering mechanism. This effectively solves the problem of industrial numerical storage and the extraction of numbers with a decimal point. In the experimental results, for both Google voice delivery and human voice delivery, the proposed IRAKE method of keyword extraction improves the accuracy rate of the original RAKE method by $16 \%$. Because the amount of voice data is small, statistical methods are used. When the amount of collected voice data is large enough, deep learning methods can be used in future work to directly implement voice commands to control the robotic arm.

\section{References}

1 M. Madankar, M. B. Chandak, and N. Chavhan: Procedia Comput. Sci. 78 (2016) 84. https://doi.org/10.1016/j. procs.2016.02.071

2 K. F. McCoy, J. L. Arnott, L. Ferres, M. Fried-Oken, and B. Roark: Comput. Speech Lang. 27 (2013) 1143. https://doi.org/10.1016/j.csl.2013.04.005

3 Q. Jiangtao, L. Chuanhui, L. Yinghong, and L. Zhangxi: Inf. Sci. 451-452 (2018) 295. https://doi.org/10.1016/j. ins.2018.04.009

4 R. Errattahi, A. E. Hannani, and H. Ouahmane: Procedia Comput. Sci. 128 (2018) 32. https://doi.org/10.1016/j. procs.2018.03.005

5 C. H. You and B. Ma: Speech Commun. 94 (2017) 30. https://doi.org/10.1016/j.specom.2017.08.007

6 K. Vani and D. Gupta: Inf. Process Manag. 54 (2018) 408. https://doi.org/10.1016/j.ipm.2018.01.008

7 C. Kennington and D. Schlangen: Comput. Speech Lang. 28 (2014) 240. https://doi.org/10.1016/j.csl.2013.06.004

8 X. Wang and C. Yuan: CAAI Trans. Intell. Technol. 1 (2016) 303. https://doi.org/10.1016/j.trit.2016.12.004

9 C. Kennington and D. Schlangen: Comput. Speech Lang. 28 (2014) 240. https://doi.org/10.1016/j.csl.2013.06.004

10 J. Wang, J. Su, and Y. Xi: Inf. Fusion. 2 (2001) 261. https://doi.org/10.1016/S1566-2535(01)00042-2

11 J. Savage, A. L. Larena, G. Carrera, S. Cuellar, D. Esparza, Y. Minami, and U. Penuelas: Lecture Notes in Computer Science, U. Visser, F. Ribeiro, T. Ohashi, and F. Dellaert, Eds. (Springer, Berlin, Heidelberg, 2007) pp. 512-519.

12 M. Rada and T. Paul: Proc. 2004 Conf. Empirical Methods in Natural Language Processing (2004) 404. https:// www.aclweb.org/anthology/W04-3252

13 G. S. Kumar and G. Zayaraz: Comput. Inf. Sci. 27 (2015) 13. https://doi.org/10.1016/j.jksuci.2014.03.001

14 A. Mohasseb, M. Bader-El-Den, and M. Cocea: Inf Process Manag. 54 (2018) 1228. https://doi.org/10.1016/j. ipm.2018.05.001

15 S. Rose, D. Engel, N. Cramer, and W. Cowley: Text Mining: Applications and Theory, M. W. Berry and J. Kogan, Eds. (John Wiley \& Sons, Ltd, Chichester, UK, 2010) pp. 3-20.

16 T. Shouzhong and H. Minlie: J. China Univ. Posts Telecommun. 23 (2016) 40. https://doi.org/10.1016/S1005$\underline{8885(16) 60056-0}$ 


\section{About the Authors}

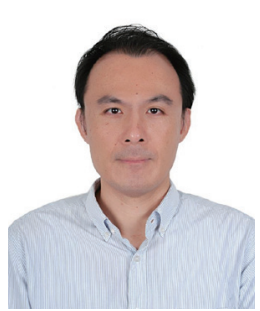

Chi-Huang Shih received his Ph.D. degree in electrical engineering from National Cheng Kung University, Taiwan, R.O.C., in 2008. Currently, he is an associate professor of the Computer Science and Information Engineering Department, National Chin-Yi University of Technology, Taichung, Taiwan, R.O.C. His current research interests are machine learning, multimedia network communication, biomedical worn devices, embedded systems, and IoT applications.

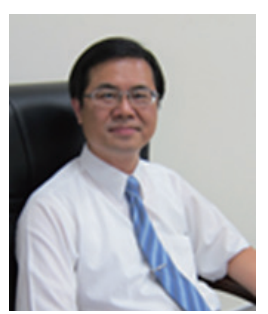

Cheng-Jian Lin received his B.S. degree in electrical engineering from Ta Tung Institute of Technology, Taipei, Taiwan, R.O.C., in 1986, and his M.S. and $\mathrm{Ph} . \mathrm{D}$. degrees in electrical and control engineering from National Chiao-Tung University, Taiwan, R.O.C., in 1991 and 1996, respectively. Currently, he is a chair professor of the Computer Science and Information Engineering Department, National Chin-Yi University of Technology, Taichung, Taiwan, R.O.C., and dean of Intelligence College, National Taichung University of Science and Technology, Taichung, Taiwan, R.O.C. His current research interests are machine learning, pattern recognition, intelligent control, image processing, intelligent manufacturing, and evolutionary robots. (cjlin@ncut.edu.tw)

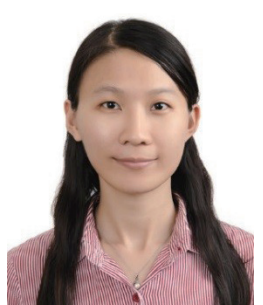

Shiou-Yun Jeng received her Ph.D. degree in industrial engineering and management from National Yunlin University of Science \& Technology, Taiwan, R.O.C., in 2019. Currently, she is an assistant professor of the Business Administration Department, Asia University, Taichung, Taiwan, R.O.C. Her current research interests are artificial intelligence, fuzzy theory, sustainable supply chain management, resource efficiency, green production management, and big data analysis. 